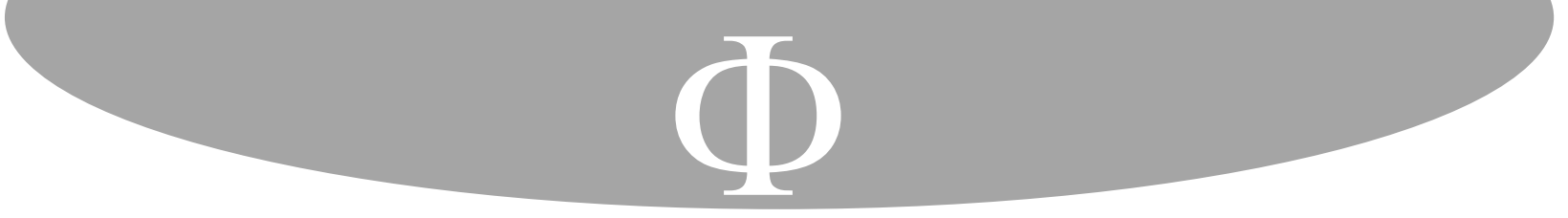

\title{
Un criterio filosófico de orientación educativa a partir de la idea de ingenio de Juan Huarte
}

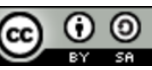

\author{
Cristián Alejandro De Bravo Delorme* \\ Universidad de Sevilla \\ Sevilla, España
}

Para citar este artículo: De Bravo Delorme, Cristián Alejandro. «Un criterio filosófico de orientación educativa a partir de la idea de ingenio de Juan Huarte». Franciscanum 176, Vol. 63

(2021): 1-28.

\section{Resumen}

El siguiente artículo propone un análisis de la idea de ingenio de Juan Huarte para la construcción de un criterio filosófico que oriente al individuo a elegir la ciencia que más le corresponda. Este criterio, según Huarte, no se orienta por los gustos o deseos de los individuos, sino por las inclinaciones naturales objetivadas por las capacidades ejercidas. En contraste con un enfoque psicológico, el criterio de Huarte se presenta como un criterio fundado en ideas filosóficas, las cuales se consideran dentro de un sistema de pensamiento que coordina las potencias racionales del individuo con relación a las ciencias, artes y técnicas correspondientes a los distintos ingenios.

\section{Palabras clave}

Huarte, ingenio, orientación, educación, filosofía.

\section{A philosophical criterion of educational guidance from the idea of wit by Juan Huarte}

\begin{abstract}
The following paper offers an analysis of the idea of wit according to Juan Huarte in order to construct a philosophic criterion that guides the individual to choose the science that best corresponds to them. This criterion is based on the determination of the different types of wit for the sciences and, according to Huarte, it is not guide by likes or wishes of the individual, but by the natural propensities objectified by the exercised capacities. In contrast to a psychologic perspective, Huarte's criterion represents an approach based on philosophic ideas, which are deemed within a system of thought that coordinates the intellectual faculties in relation to the sciences, arts and techniques corresponding to the different types of wits.
\end{abstract}

\footnotetext{
* Doctor en filosofía. Es Asistente Honorario del Departamento de Estética e Historia de la Filosofía de la Facultad de Filosofía de la Universidad de Sevilla y sus líneas de investigación son: fenomenología, hermenéutica y filosofía griega. ORCID: https://orcid.org/0000-0002-6363-9165. Contacto: debravo.cristian@gmail.com; cdebravo@us.es.
} 


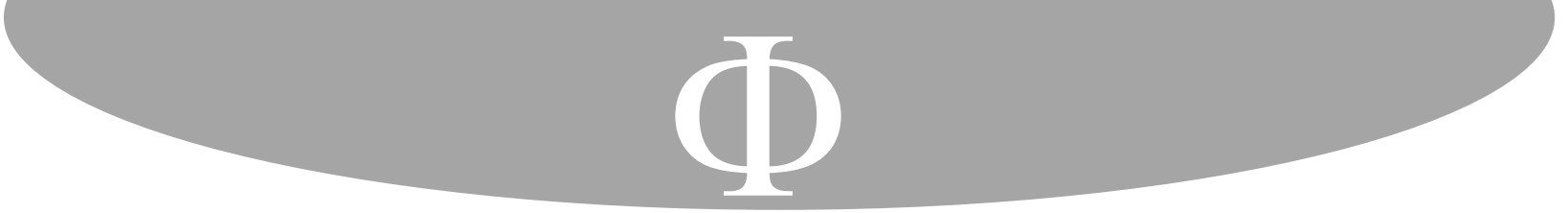

\section{Keywords}

Huarte, ingenuity, orientation, education, philosophy.

\section{Consideraciones preliminares}

Desde que la Ley General de Educación de 1970 contempló la existencia de servicios de orientación pedagógica dentro de los centros escolares, se ha vuelto cada vez más evidente la relevancia de la orientación del alumnado dentro del sistema educativo español ${ }^{1}$. En la medida que la enseñanza reglada ha llegado a ser un proceso mediante el cual los individuos no sólo adquieren conocimientos y destrezas, sino las herramientas para desarrollar su personalidad, la orientación educativa ha pasado de ser una estructura marginal a ser un pilar fundamental de la educación de los jóvenes. En correspondencia con ello, el docente ha debido incorporar a su labor educativa la acción orientadora, complementando de esta manera su labor de instrucción con la necesaria función de tutor del alumno ${ }^{2}$.

La orientación educativa, en términos generales, podría determinarse como un conjunto de conocimientos, metodologías y principios teóricos que dan base a una intervención de corte psicopedagógico dirigida al desarrollo integral de los individuos ${ }^{3}$. Este desarrollo integral con frecuencia se ha definido a la luz de la vocación individual, por lo que cabe afirmar que la orientación tiene como uno de sus fines más importantes que el individuo sea capaz de responder a su «llamada». En este sentido, no sólo la correcta elección profesional, sino, más importante aún, la realización de la propia persona dependería de «escuchar» correctamente esta vocación ${ }^{4}$. La orientación educativa con base en el descubrimiento, desarrollo y la madurez vocacional ${ }^{5}$ determinaría así un criterio psicológico por el cual el individuo, bajo el cuidado de tutores y familiares, se proveería de las coordenadas suficientes para dirigir su propia trayectoria vital.

\footnotetext{
${ }^{1}$ Manuel Álvarez, Orientación profesional (Barcelona: Cedecs, 1995); María Luisa Rodríguez, La orientación profesional (Barcelona: Ariel, 1998); Alfredo Pérez y Pilar Blasco, Orientación e inserción profesional: Fundamentos y tendencias (Valencia: Nau Llibres, 2003); Andrés Negro, La orientación en los centros educativos: Organización y funcionamiento desde la práctica (Barcelona: Graó, 2006); Antonia Parras, Ana María Madrigal, Sara Redondo, Patricia Vale, \& Enrique Navarro, Orientación educativa: fundamentos teóricos, modelos institucionales y nuevas perspectivas (Ministerio de Educación: CIDE, 2009); Pablo Ormaza Mejía, «Orientación vocacional y profesional», Revista Ciencia UNEMI 30, Vol. 12 (2019): 87-102.

${ }^{2}$ Víctor Álvarez-Rojo, Orientación educativa y acción orientadora: Relaciones entre la teoría y la práctica (Madrid: EOS, 1994); Francisco Imbernón, coord., Procesos y contextos educativos: Enseñar en las instituciones de educación secundaria (Barcelona: Graó, 2010).

${ }^{3}$ Consuelo Veláz de Medrano, Orientación e intervención psicopedagógica: Concepto, modelos, programas y evaluación (Archidona: Aljibep, 1998), 37.

${ }^{4}$ Jesús Busot, Elección y Desarrollo Vocacional (Caracas: Ediluz, 1995). No resulta impertinente en este punto advertir la raigambre teológica de la vocación. Véase Mt. 33, 14: multi autem sunt vocati pauci vero electi. Septuaginta, editada por Alfred Rahlfs (Stuttgart: Deutsche Bibelgesellschaft, 1979).

${ }^{5}$ Donald Super, «Dimensions and measurements of vocational maturity», Teachers college records 3, Vol. 57 (1955): 151-163.
} 


\section{La idea de ingenio de Juan Huarte ${ }^{27}$}

Que Juan Huarte considere que el ingenio se constituye con base en el temperamento no resulta algo original, pues antes de publicarse el Examen de ingenios Juan Luis Vives, quien se apoya tal como Huarte en toda la tradición hipocrático-galénica, ya había señalado que «el calor y los humores debidamente combinados, contribuyen a la agudeza y salud del ingenio ${ }^{28}$. Pero si bien Vives y mucho antes Galeno, de cuyo pensamiento Huarte se declara deudor, no desconocen que el ingenio depende del temperamento, ninguno tematiza las diferencias temperamentales en función de los ingenios para las ciencias. De ahí que, según Huarte, se requiera un examen crítico de las diferencias de ingenio que pueda orientar a los tutores de la república a interpretar las inclinaciones naturales de los estudiantes ${ }^{29}$. Con esto se anuncia, además, que el propio tutor debe contar con un determinado ingenio que lo capacite para la tarea de detectar el ingenio correspondiente a cada individuo.

Para el presente artículo no importa tanto la doctrina de los humores que Juan Huarte hereda de la tradición filosófico-médica. Sin embargo, no cabe soslayar la necesidad de comprender orgánicamente las potencias y operaciones racionales, por lo que vale mencionar la insistencia de Huarte en la determinación del temperamento del cerebro como base orgánica del ingenio y, a su vez, en el tipo de clima en el que alguien nació, así como su dieta, el agua que bebe y todo lo relativo a su régimen de vida ${ }^{30}$. Esto resulta importante, porque del temperamento se engendran las inclinaciones naturales y entre ellas los ingenios adecuados para aprender ciertas ciencias, técnicas o artes, que son disposiciones y hábitos ${ }^{31}$. Lo que ante todo importa en este contexto es que las determinadas proporciones de las calidades humorales, que tradicionalmente eran cuatro y que Huarte reduce a tres por estimar que una de ellas tiene sólo una función reguladora ${ }^{32}$, se encuentran coordinadas según una

\footnotetext{
${ }^{27}$ Para un estudio completo de la vida y obra de Huarte véase Mauricio Iriarte, El Doctor Huarte de San Juan y su «Examen de ingenios»: contribución a la historia de la psicología diferencial (Madrid: Consejo Superior de Investigaciones Científicas, 1948).

${ }^{28}$ José Luis Vives, El alma y la vida. Biblioteca valenciana digital, consultada el 9 de julio, 2020, consultada en julio 9, 2020, https://bv2.gva.es/corpus/unidad.do?idCorpus=1\&idUnidad=9986\&posicion=1.

${ }_{29}^{29}$ «Huarte estaba persuadido de algo fundamental para él, a saber, el hecho diferencial de la inteligencia, o de las diferencias de ingenio, como él las llamaría, y el hecho diferencial de las profesiones académicas, que requerían habilidades muy distintas en quienes las practicaban. La naturaleza nos había hecho diferentes y ello significaba que no estábamos igualmente capacitados para todas las profesiones, debiendo elegir la que mejor se adecuara a nuestro talento natural». José María Gondra, «Juan Huarte de San Juan y las diferencias de inteligencia», Anuario de Psicología Vol. 69, 13-34 (1994): 16. Cabe señalar que la idea de las diferencias de ingenio proviene de Platón o, al menos, es en los diálogos platónicos donde por primera vez se hace referencia a la correspondencia entre un oficio y un determinado talento. Platón, República, 370a-b, en Diálogos, Vol. 1, trad. C. García Gual y F. J. Olivieri y E, Lledó (Madrid: Gredos, 2011).

${ }^{30}$ Juan Huarte, Examen de ingenios, 246.

${ }^{31}$ En términos por los cuales cabe comparar la cultura animi con la agricultura Huarte afirma: «Y así dice Hipócrates que el ingenio del hombre tiene la misma proporción con la ciencia que la tierra con la semilla; la cual, aunque sea de suyo fecunda y paniega, pero es menester cuidarla y mirar para qué género de simiente tiene más disposición natural. Porque no cualquier tierra puede panificar con cualquiera simiente sin distinción: unas llevan mejor trigo que cebada, y otras mejor cebada que trigo, y del trigo tierras hay que multiplican mucho candial y el Trujillo no lo pueden sufrir». Juan Huarte, Examen de ingenios, 224-225.

32 «Pero de cuatro calidades que hay (calor, frialdad, humedad y sequedad) todos los médicos echan fuera la frialdad por inútil para todas las obras del ánima racional...sólo sirve en el cuerpo de templar en calor natural
} 


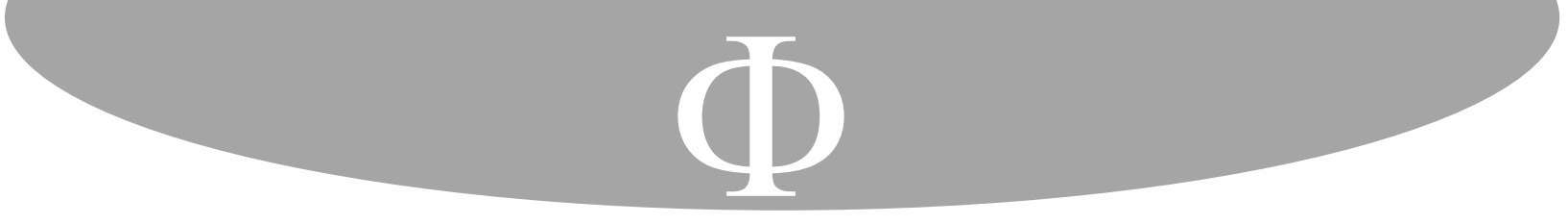

de San Agustín y, con mayor precisión, el esquema ternario de Juan Huarte. Huelga decir que la novedad en estos casos no es sino un olvido del pasado.

Pues bien, consideremos en primer lugar que Juan Huarte, como buen médico que era $^{39}$, comienza su examen declarando por experiencia que distintos individuos responden de manera diferente a las mismas enseñanzas. Así constata que, en un grupo de tres jóvenes, uno que responde mal al aprendizaje de una lengua, otro, en cambio, la aprende con ligereza (memoria). Mientras a éste, sin embargo, se le da mal el arte de argumentar, el primero puede discurrir con soltura y arte (entendimiento). El tercero, por otro lado, que no puede ni con las lenguas ni con la dialéctica, puede con natural talento manejar números y fórmulas matemáticas (imaginación). Huarte, por lo tanto, infiere que «las ciencias eran como naturales a solos los hombres que tienen ingenios acomodados para ellas». ${ }^{40}$ Huarte, por lo tanto, considera necesario distinguir las verdaderas señales con que se descubren los ingenios para que la función que desempeñe el individuo en sociedad sea objetivamente fértil y subjetivamente satisfactoria.

En la versión de 1594 de su Examen de ingenios, Huarte afirma, basado en una tradición que cabe remontar a Platón ${ }^{41}$, que se pueden distinguir en el hombre dos potencias generativas; una que es común a los animales y plantas, y otra propia del hombre. Esta potencia generativa propia del hombre es afín a la potencia divina y, afirma Huarte, vale comprenderla en analogía con las dos personas de la trinidad, pues tal como Dios engendra al Hijo, el entendimiento genera el concepto (verbum mentis). De ahí, dice Huarte, que por su gran fecundidad se denomine a Dios Genio, «que por antonomasia quiere decir el grande engendrador ${ }^{42}$. La fecundidad del hombre, sin embargo, es claramente distinta a la divina, no sólo porque el engendro conceptual que nace del entendimiento no es un «ser real y sustantífico fuera de sí» (puesto que es un accidente producido en la memoria y no más que «una figura y retrato de aquello que queremos saber y entender»), sino porque cuando implica un producto fabricado «es menester fingir primero mil rayas en el aire, y componer muchos modelos, y últimamente poner las manos para que tomen el ser que han de tener, y las más veces salen erradas ${ }^{43}$. Huarte señala que el genio humano genera el concepto, que en relación

\footnotetext{
${ }^{39}$ En relación con el contexto médico del pensamiento de Huarte véase José Luis Orella, «El pensamiento filosófico y médico de Juan Huarte», Sancho el sabio: Revista de cultura e investigación vasca 6 (1996), 57 y ss. y Carlos Noreña, Studies in Spanish Renaissance Thought (The Hague: Martinus Nijhoff, 1975), 210 y ss.

${ }^{40}$ Juan Huarte, Examen de ingenios, 221. Cabe en este sentido apuntar que la tesis según la cual al individuo le corresponde una ciencia o arte en función de su ingenio, viene de reconocer, por una parte, la igualdad natural del ánima racional y, por otra parte, las diferencias de ingenio debido a su carácter orgánico. «[S]i el entendimiento estuviese apartado del cuerpo y no tuviese que ver con el calor, frialdad, humedad y sequedad, ni con las demás calidades corporales, se seguiría que todos los hombres tendrían igual entendimiento y que todos raciocinarían con igualdad. Y vemos, por experiencia, que un hombre entiende mejor que otro y discurre mejor. Luego ser el entendimiento potencia orgánica, y estar en uno más bien dispuesta que en otro, lo causa; y no por otra razón ninguna. Porque todas las ánimas racionales y sus entendimientos, apartados del cuerpo, son de igual perfección y saber». Juan Huarte. Examen de ingenios, 355-356.

${ }^{41}$ En el Banquete, Diótima le dice a Sócrates: «Impulso creador, Sócrates, tienen, en efecto, todos los hombres, no sólo según el cuerpo, sino también según el alma». Platón, Diálogos I, 206c.

42 Juan Huarte, Examen de ingenios, 189.

${ }^{43}$ Juan Huarte, Examen de ingenios, 191.
} 


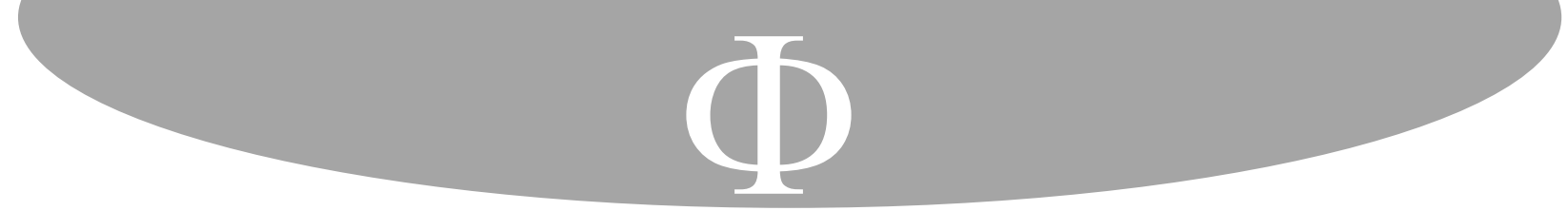

Según lo dicho, la imaginación podría decirse que es el primer analogado de la idea de ingenio, puesto que el ingenioso se estima en gran medida por ser inventivo y creador, y las acciones y obras relativas a la potencia imaginativa se denominan, en este sentido, mayormente ingeniosas. Si el memorioso tiene como característica la retención de las figuras y conceptos y el inteligente el juicio de las cosas presentes, el imaginativo se encuentra referido a «lo que está por venir» ${ }^{60} \mathrm{y}$, en este sentido, la imaginación acompaña a la prudencia, pues precisamente la prudencia como potencia práctica se atiene a lo que puede ser. Este tipo de ingenio puede variar de muchas maneras, pues en la medida que está referido a lo que estrictamente no es, funciona con base en posibilidades, lo cual requiere destreza de ánimo y, como declara Huarte, agudeza, solercia, astucia, cavilo y engaños. El imaginativo es diestro en la elocuencia, la gracia y la presteza en el hablar y responder a propósito. De ahí que la imaginación descubra «todas las virtudes y vicios del hombre porque en cada momento se ofrecen ocasiones en las cuales da el hombre muestras de lo que haría en otras cosas mayores» ${ }^{61}$.

Huarte declara con agudeza que precisamente en este tipo de ingenio, cuya potencia mayor es la imaginación y que en cuanto potencia relativa al futuro y asociada a la prudencia ética se encuentra presente en menor proporción en los otros ingenios, manifiesta los hábitos de los individuos, pues cuando se trata del comercio con los otros hombres se expresan costumbres, pero también estrategias de comportamiento. Dicho de otra manera, en la medida que entre seres humanos la verdad se vuelve un asunto mezclado con la apariencia, la imaginación delata tanto las obras claras como las solapadas, pero también las ironías y las acciones que muchos, según Huarte, no sin razón asocian a lo «demoníaco» en el sentido de lo extraordinario y portentoso ${ }^{62}$.

En este contexto Huarte puede afirmar que la imaginación, en la medida que se encuentra asociada al calor temperamental, puede avivarse en cualquiera que sufra de tal excitación, de manera que el ingenio imaginativo puede potenciarse, pero también exacerbarse, deviniendo manía, como aquella de la que habla Sócrates en el Fedro ${ }^{63}$ o bien patología y, en último término, enfermedad. De ahí que el imaginativo se encuentre muy próximo a la destemplanza, por un exceso de calor que, como en el caso de Don Quijote, le produjo sequedad extrema en el cerebro al punto de exacerbar su imaginación por tanta lectura de libros de ficción ${ }^{64}$.

Con todo, este ingenio que pone de relieve la imaginación como potencia eminente hace a los individuos creativos $\mathrm{y}$, al extremo, inventivos por caprichosos. Así lo indica la lengua toscana, señala Huarte, pues este ingenio es semejante a esa cabra que «es amiga de

\footnotetext{
${ }^{60}$ Juan Huarte, Examen de ingenios, 373.

${ }^{61}$ Juan Huarte, Examen de ingenios, 408.

${ }^{62}$ Juan Huarte, Examen de ingenios, 304-320.

${ }^{63}$ Platón, Diálogos I, 2011a, 244a y ss.

${ }^{64}$ Véase Rafael Salillas, Un gran inspirador de Cervantes, el doctor Juan Huarte y su examen de ingenios (Pamplona, Navarra: Analecta, 2003).
} 


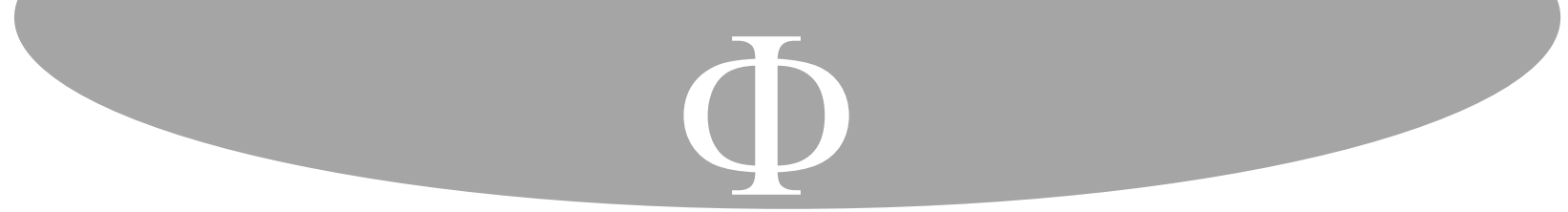

lo que parece cosa imposible. De tal manera, que si al médico le preguntásemos cómo pudo atinar a conocimiento tan delicado, no sabría dar la razón; porque es gracia que nace de una fecundidad de la imaginativa que por otro nombre se llama solercia, la cual con señales comunes, inciertas, conjeturales y de poca firmeza, en cerrar y abrir el ojo alcanzan mil diferencias de cosas en las cuales consiste la fuerza del curar y pronosticar con certidumbre ${ }^{75}$.

Huarte no atribuye a la imaginación una suerte de irracionalidad, lo cual sería ridículo, considerando que la imaginación es parte de la razón, pero tampoco le atribuye algún poder místico que superase a la propia razón. Más ridículo sería esto, si estimamos la formación médica de Huarte y su apelación constante a la filosofía natural como ciencia pertinente para comprender lo que sea el ingenio. Huarte a su modo está indicando la estructura originaria de la racionalidad, cuya memoria y discurso necesitan previamente de la visión de la imaginación, la cual se extiende en los sentidos externos. De ahí que Huarte confirme que esta visión se acredita en el diagnóstico de los mejores médicos, quienes, con tan sólo sentir el pulso del enfermo, ver su aspecto, escuchar su respiración y oler su orina, atinan con la enfermedad y con el consecuente tratamiento. Y así, recordando a Galeno, Huarte señala que al médico se le conoce por estas razones como un inventor occasionis, por inventar el tiempo, el lugar y la ocasión ${ }^{76}$. Pero aquí inventor no quiere decir tanto el que fabrica o arma algún artilugio como el que descubre, de manera que la invención del médico consiste en dejar venir a las cosas para que se muestren a sí mismas, desvelarlas y así darles una figura y correspondencia.

Lo más interesante de lo señalado por Huarte estriba en que el médico pone de relieve la imaginación en su más original función y que, en términos generales, consiste en dar lugar y dar tiempo. Lo que el médico acredita es una potencia que da lugar a que la enfermedad se revele y da tiempo para el tratamiento necesario para recuperar la salud. El pleno ejercicio del médico, sin embargo, resulta muy raro, pues Huarte le atribuye las tres potencias que difícilmente pueden operar en conjunto de manera proporcional en la mayoría de los individuos. Para los efectos de esta investigación es importante advertir que la imaginación se revela de tal modo para el juicio del orientador, que cabe asociarla a todas aquellas ciencias, artes y técnicas que requieran precisamente de una atinada manera de ser con relación a las cosas.

Más aún, es necesario advertir que, si bien el pleno ejercicio de la imaginación en conjunto con las otras potencias es escaso, no por ello resulta innecesario ejercitar la imaginación en todos los estudiantes. Esto es del todo relevante, aunque no tan sólo para que los correspondientes jóvenes puedan desarrollar sus habilidades en el dibujo, en las destrezas manuales o bien en la práctica de ciencias como la medicina, sino, además, para desarrollar la propia persona.

\footnotetext{
75 Juan Huarte, Examen de ingenios, 500-501.

${ }^{76}$ Juan Huarte, Examen de ingenios, 502.
} 
